\title{
Zur Reproduzierbarkeit der Wegstrecken beim 6-Minuten-Gehtest im Rahmen eines stationären Rehabilitationsaufenthaltes
}

\author{
A New Procedure for the Estimation of Physical Fitness of Patients During \\ Clinical Rehabilitation using the 6-Minutes Walk Test
}

Autoren

Institute
W. Marek ${ }^{1}$, E. Marek ${ }^{1,4}$, Y. Friz' ${ }^{1}$ P. Vogel ${ }^{3}$, K. Mückenhoff ${ }^{2}$, N. Kotschy-Lang ${ }^{3}$

Institut für Arbeitsphysiologie an der Augusta-Kranken-Anstalt, Bochum

2 Institut für Physiologie, Ruhr-Universität-Bochum, Bochum

Berufsgenossenschaftliche Klinik für Berufskrankheiten Falkenstein, Falkenstein

${ }^{4}$ Institut für Sportmedizin und Sporternährung, Ruhr-Universität Bochum, Bochum eingereicht 27.5. 2009

akzeptiert nach Revision 9. 9.2009

\section{Bibliografie}

Dol http://dx.doi.org/

10.1055/s-0029-1215233

Online-Publikation: 12. 1. 2010

Pneumologie 2010; 64:

155-162 @ Georg Thieme

Verlag KG Stuttgart · New York

ISSN 0934-8387

\section{Korrespondenzadresse \\ PD Dr. rer. nat. \\ Wolfgang Marek}

Institut für Arbeitsphysiologie an der Augusta-Kranken-Anstalt Bergstr. 26

Bochum

Wolfgang.Marek@ruhr-unibochum.de

\section{Zusammenfassung $\nabla$}

Ziel der Untersuchung: Der bei älteren Patienten durchgeführte 6-Minuten-Gehtest (6 MWT) wird häufig wiederholt, um die maximale Gehstrecke zu dokumentieren, obwohl dies nicht ausdrücklich in den Richtlinien zur Durchführung von Belastungstests empfohlen wird. Die Wiederholungen erfordern Zeit und einen hohen Personalaufwand. Auf der anderen Seite gibt es eine vernünftige Forderung, mit diesen klinischen Ressourcen sparsam umzugehen. Wir überprüften daher, ob die Durchführung des Gehtestes ohne Wiederholung hinreichende und verlässliche Information über die körperliche Leistungsfähigkeit der Patienten und deren Veränderung im Laufe ihres Rehabilitationsaufenthaltes geben würde.

Methoden: 35 Patienten absolvierten ihren Gehtest zu Beginn und zum Ende einer 3-4-wöchigen klinischen Therapie. Jeder Test wurde jeweils nach einer Erholungszeit von einer Stunde wiederholt. Die Patienten wurden aufgefordert, während einer Zeit von 6 Minuten so schnell wie möglich zu gehen. Sie waren mit einem mobilen Pulsoximeter zur Messung der Sauerstoffsättigung des Blutes und der Herzfrequenz ausgestattet. Gemessen wurden: Der Gehweg, S, die Herzfrequenz, fc, und die Effizienz, E =S/6/fc, werden als Quotient aus der in einer Minute zurückgelegten Gehstrecke in Metern und der mittleren Herzfrequenz während der Belastungsphase berechnet.

Ergebnisse: Im ersten Test gingen die Patienten eine Gehstrecke von $416 \pm 63 \mathrm{~m}$ mit einer Herzfrequenz von 104,7 $\pm 15,7 / \mathrm{min}$; im ersten wiederholten Test: $454 \pm 71 \mathrm{~m}$ mit einer Herzfrequenz von 106,3 $\pm 17,4 / \mathrm{min}$. Im ersten Abschlusstest, nach klinischer Therapie, erreichten sie 438 $\pm 58 \mathrm{~m}$ mit einer Herzfrequenz von $106,3 \pm 17,4$ / min; im wiederholten Test: $473 \pm 56 \mathrm{~m}$ mit einer Herzfrequenz von $108,6 \pm 13,2 / \mathrm{min}$. Die sich ergebenden Gehwegdifferenzen, 38,4 $\pm 26,2 \mathrm{~m}(+9,3$ $\pm 6,2 \%)$ zu Beginn und $35 \pm 26 \mathrm{~m}(+8,6 \pm 6,4 \%)$

\section{Abstract \\ $\nabla$}

Aims of the investigation: The repetition of the 6-minutes walk test (6 MWT) in older patients is frequently performed in order to document the maximal walking distance, although it is not recommended in any guidelines on exercise tests and although there is common consent to save clinical resources in terms of time and staff. Therefore, we have examined whether and to what extent the repetition of the walk tests helps patients to get more familiar with this kind of exercise test. Thus the acquired physiological data should reliably describe the physical fitness of the patients at the beginning and at the end of their clinical rehabilitation.

Methods: 35 patients performed their walk tests before and after 3-4 weeks of clinical rehabilitation. Each test has been repeated after one hour of recovery. The patients were instructed to walk during 6 minutes as fast as possible. They were equipped with a mobile pulse oximeter for recording oxygen saturation and heart rate. The distance, $\mathrm{S}$, and the heart rate, fc, were measured. Measurements were performed every 30 seconds and recorded. The efficiency, $E(E=S / 6 / f c)$, was calculated as the ratio of distance per minute and the mean heart rate during the test.

Results: In the first test the patients walked 416 $\pm 63 \mathrm{~m}$ at a heart rate of $104.7 \pm 15.7$ beats $/ \mathrm{min}$, in the first repeated test $454 \pm 71 \mathrm{~m}$ at a heart of $106.3 \pm 17.4$ beats $/ \mathrm{min}$. In the second test, after clinical therapy, they walked $438 \pm 58 \mathrm{~m}$ at a heart rate of $106.3 \pm 17.4$ beats $/ \mathrm{min}$, in the second repeated test $473 \pm 56 \mathrm{~m}$ at $108.6 \pm 13.2 / \mathrm{min}$. The difference of the walking distances of the tests at the entrance were found to be $38.4 \pm 26.2 \mathrm{~m}(+9.3$ $\pm 6.2 \%$ ), at the end of clinical rehabilitation 35 $\pm 26 \mathrm{~m}(+8.4 \pm 6.4 \%)$. Both differences are found to be independent from the distance of the first test. They are not significantly different. The efficiency was not significantly different in the initial 
zum Ende sind nicht signifikant verschieden: sie sind unabhängig von der Gehstrecke im jeweilig ersten Test. Es wurden die folgenden Effizienzen, E, gemessen: 0,673 $\pm 0,129$ zu Beginn und 0,689 $\pm 0,085 \mathrm{~m} / \mathrm{HS}$ zum Abschluss der Rehabilitationsmaßnahme. Die folgenden Abweichungen der Effizienzen zwischen Eingangsund Wiederholungstest wurden berechnet: 0,053 \pm 0,062 zu Beginn und 0,042 $\pm 0,047$ am Ende der Rehabilitation. Beide sind nicht signifikant verschieden.

Schlussfolgerungen: Sowohl im Eingangstest als auch im Abschlusstest führte die Wiederholung zu signifikanten, aber vergleichbaren Steigerungen der Wegstrecke und der Effizienz. Die Patienten erreichten dabei eine im Mittel um 1-2 Herzschläge/ min höhere Pulsfrequenz. Dieses deutet auf eine nur kurz andauernde Bereitschaft der Patienten hin, sich einer höheren körperlichen Belastung auszusetzen, die über die Zeit der Dauer der Rehabilitation nicht anhält. Daher kann in der klinischen Routine auf die Wiederholungen des Eingangs- und des Ausgangstestes verzichtet werden. Der Parameter Effizienz ist eine sinnvolle Ergänzung zur ausschließlichen Betrachtung des zurückgelegten Weges. Er objektiviert die körperliche Leistungsfähigkeit bzw. den Rehabilitationserfolg auch dann, wenn der Patient am Ende einer Rehabilitationsmaßnahme eine kürzere Wegstrecke zurücklegt.

\section{Einleitung}

$\nabla$

Ein einfaches und kostengünstiges Verfahren, um die körperliche Leistungsfähigkeit älterer Probanden und damit einen bedeutenden Bestandteil ihrer Lebensqualität zu bestimmen, ist der 6-Minuten-Gehtest (6 MWT) [1]. Unter Anleitung wird die Gehstrecke (6 MWD) bestimmt, die der Proband in einer Zeit von 6 Minuten maximal zurücklegt [2]. Der Test ermöglicht einerseits das Ausmaß täglicher Aktivitäten abzuschätzen und andererseits funktionelle Einschränkungen zu finden. Standardisierungen der Methode erwägen eine Wiederholung des Tests, um die maximal erreichbare Wegstrecke zu bestimmen [3-7], so auch die Deutsche Gesellschaft für Pneumologie [8]. Es stellt sich die Frage nach dem Lerneffekt durch eine ökonomischere Belastung bei der Wiederholung des 6 MWT und damit verbunden die Frage, ob dieser Lerneffekt nach 3-4 Wochen Rehamaßnahme noch vorhanden ist. Der 6 MWT kann auch von älteren und gebrechlichen Menschen oder stärker eingeschränkten Patienten, die nicht mit der Standardmethode der Fahrrad- oder Laufbandergometrie getestet werden können, bewältigt werden $[9,10]$. Der 6 MWT ist auch in der berufsgenossenschaftlichen Klinik für Berufskrankheiten Falkenstein eines der wesentlichen Testverfahren zur Objektivierung der körperlichen Leistungsfähigkeit von älteren Patienten im Rahmen eines stationären Rehabilitationsaufenthaltes. Er wird zu Beginn und am Ende des stationären Aufenthaltes durchgeführt. Unter Anleitung werden die Patienten aufgefordert, in 6 Minuten die für sie maximal mögliche Gehstrecke zu absolvieren. Eine Verbesserung um mehr als $54 \mathrm{~m}$ wird dabei als klinisch relevante Steigerung angesehen. Dies wird aus unterschiedlichen Gründen jedoch nur von einem Teil der Patienten erreicht, sodass weitere Parameter für die Beurteilung der körperlichen Leistungsfähigkeit bzw. den Erfolg einer Rehabilitationsmaßnahme wünschenswert sind. Bei verbesserter körperlicher Leistungsfähigkeit, wie sie im Rahmen der Rehabilitationsmaßnahme angestrebt wird, bleibt der lineare Zusammenhang zwischen Gehstrecke und Herzfrequenz erhalten, die Kennlinie verläuft jedoch flacher. Wir überprüften daher an einem reprä- and final test $(0.673 \pm 0.129$ and $0.689 \pm 0.085 \mathrm{~m} /$ beat, respectively). The difference in efficiency, when repeating the tests at the beginning, was: $0.053 \pm 0.062 \mathrm{~m} /$ beat; at the end of the rehabilitation: $0.042 \pm 0.047 \mathrm{~m} /$ beat. They are found to be similar.

Conclusions: The distances the patients walked in the repeated tests at the entrance and at the end of their clinical rehabilitation were, besides the calculated efficiency, E, significantly increased. However, the increases in distance and efficiency are identical on both occasions, therefore the repetition delivers no further information. The test should be performed without repetitions in clinical routine investigations. The patient's performance in the second walk test with an unchanged distance at a lower heart rate reveals an improved physical fitness. This is solely described by an increase of efficiency, E. Therefore the introduction of $\mathrm{E}$ is a suitable measure of the quantified effect of exercise training, even if the patient is not cooperative during the tests. $E$ is proved to be a suitable estimation for the assessment of physical fitness as a benefit of clinical rehabilitation. sentativen Patientengut der Klinik, ob die Wiederholungen des Gehtests jeweils zu Beginn und am Ende einer Rehamaßnahme zu weiteren Ergebnissen führen als ein einmaliger Test und ob der Lerneffekt durch die Wiederholung am Ende der Rehamaßnahme noch vorhanden ist.

\section{Methoden \\ $\nabla$}

\section{Patientengut}

Die Wiederholungsuntersuchungen des 6-WMT wurden an 35 Patienten durchgeführt, die sich zu einer 3- bis 4-wöchigen Rehabilitationsmaßnahme in der BG-Klinik Falkenstein auf Veranlassung der Berufsgenossenschaften bzw. der Unfallkassen aufhielten. Die Probanden ( $32 \mathrm{~m}, 3 \mathrm{w}$ ) hatten bei einem mittleren Lebensalter von 66,1 $\pm 9,3$ Jahren eine Körpergröße von 172 $\pm 6,7 \mathrm{~cm}$, ein mittleres Gewicht von $80 \pm 15 \mathrm{~kg}$ und einen BMI von $27,2 \pm 5,5 \mathrm{~kg} / \mathrm{m}^{2}$. Die Zusammensetzung des untersuchten Kollektivs entsprach dem Patientengut der BG-Klinik. Bei den Patienten waren Erkrankungen der Atmungsorgane als Berufskrankheiten anerkannt: Silikose (BK 4101 der BKV), Asbestose (BK 4103 der BKV), obstruktive Atemwegserkrankung (BK 4301, 4302 der BKV) und chronische obstruktive Bronchitis und Lungenemphysem infolge Unter-Tage-Tätigkeit im Steinkohlenbergbau (BK 4111 der BKV). Die Minderung der Erwerbsfähigkeit infolge dieser Berufskrankheiten betrug überwiegend zwischen 20 und $80 \mathrm{v} . \mathrm{H}$.

9 Patienten nahmen regelmäßig $\beta$-Blocker ein, 18 einen Kombinationsspray mit $\beta$-Sympathikomimetika und einem Anticholinergikum, 5 von ihnen nur bei Bedarf und weitere 9 Patienten bekamen ein Theophyllin-Präparat. 8 Patienten nahmen keine antiobstruktiven oder kardial wirksamen Medikamente. Die medikamentöse Therapie wurde im Verlauf der Rehamaßnahme lediglich bei 9 Patienten dem aktuellen Bedarf angepasst. Bei 6 Patienten wurden inhalative Bronchodilatatoren in der Therapie ergänzt, bei 2 Patienten wurden $\beta$-Blocker abgesetzt. Bei zwei Patienten konnte die Theophyllin-Dosis reduziert werden. 
Stationäre Rehabilitation in der BG-Klinik Falkenstein

Die Patienten wurden bei Antritt des Rehaaufenthaltes einer umfassenden klinischen Diagnostik unterzogen. Dazu gehören neben der Anamnese und der körperlichen Untersuchung auch Röntgenuntersuchungen des Thorax in 2 Ebenen, eine Lungenfunktionsprüfung mit Bodyplethysmografie, Spirometrie und Fluss-Volumen-Messung, eine Blutgasanalyse in Ruhe, ein RuheElektrokardiogramm sowie ein ausführlicher laborchemischer Status. Die bestehende medikamentöse Therapie wurde gegebenenfalls dem aktuellen Gesundheitszustand angepasst. Sofern eine Sauerstoff-Langzeittherapie erforderlich war, wurde diese eingeleitet.

Ein wesentlicher Bestandteil der klinischen Rehabilitation ist die Trainingstherapie und die damit verbundene Verbesserung der körperlichen Leistungsfähigkeit und der Lebensqualität der Patienten, wie sie in den Empfehlungen der American Thoracic Society/European Respiratory Society Statement on Pulmonary Rehabilitation vorgeschlagen werden [11].

\section{6-Minuten-Gehtest}

Die Gehtests zu Beginn und am Ende des Rehaaufenthaltes wurden entsprechend den Empfehlungen der ATS/ERS [3] durchgeführt. Nach einem Erholungszeitraum von ca. 1 Stunde wurde der 6 MWT wiederholt, um einen möglichen Lerneffekt bzw. eine Ökonomisierung der Gehbelastung abschätzen zu können. Es erfolgte eine Analyse des 6 MWT von 35 Patienten, die sich zu einem 3 - 4-wöchigen Rehabilitationsaufenthalt in der Klinik Falkenstein aufhielten. Dabei wurden die Referenzwerte von Enright et al. [12] verwendet:

Sollwegstrecke $(\mathrm{m})=$

7,57 × Körpergröße - 1,76 × Gewicht - 5,02 × Alter - 309

(cm, kg, Jahre).

Der untere Grenzwert ist definiert als Sollwegstrecke $-100 \mathrm{~m}$. Für die Patienten dieser Studie entspricht eine Wegstrecke von $521 \mathrm{~m}$ dem Sollwert.

Ausgestattet mit einem mobilen Pulsoximeter (BCI 3401, USA) mit Drucker zur Registrierung der Sauerstoffsättigung, der Herzfrequenz und der Laufzeit wurden die Patienten angeleitet, die in 6 Minuten maximal zu erreichende Gehstrecke auf dem Flur der Klinik (Länge $65 \mathrm{~m}$ ) zurückzulegen und dabei regelmäßig aufgefordert, sich entsprechend zu belasten. Die Messungen erfolgten mit einem Fingerclip am Endglied eines Mittelfingers. Als Ausgangswerte („Ruhewerte“) für die Herzfrequenz und die Sauerstoffsättigung (,Ruhesättigung“) wurde der Messwert unmittelbar vor Beginn des 6 MWT verwendet (Zeit 0,0 min). Dabei erwarteten die Probanden stehend den Start zu ihrem Gehtest. Diese Messung wurde abgelesen und diente der Testleiterin zur Überprüfung der korrekten Registrierung. Die Messwerte wurden alle 30 Sekunden erhoben und ausgedruckt. Die Tests wurden unter Anleitung zu Beginn und am Ende des Rehaaufenthal- tes in der Klinik Falkenstein durchgeführt. Entsprechend den Empfehlungen für den $6 \mathrm{MWT}$ wurden die Patienten dabei ermuntert und angehalten, die Gehgeschwindigkeit ihrem Leistungsvermögen anzupassen. Ein Zugewinn an Wegstrecke von $54 \mathrm{~m}$ und mehr wird als signifikante Verbesserung der körperlichen Leistung angesehen [13].

Die körperliche Leistungsverbesserung durch eine Rehabilitationsmaßnahme wird ausschließlich durch die vergrößerte Wegstrecke infolge der Erhöhung der Gehgeschwindigkeit beschrieben. Wird der gleiche Weg unter einer niedrigeren Herzfrequenz zurückgelegt, so ist auch dies auf einen verbesserten körperlichen Trainingszustand zurückzuführen [14]. Auch eine kürzere Wegstrecke im Wiederholungstest kann bei geringerer Herzfrequenz durchaus als ein positives Ergebnis gewertet werden. Dabei dient der Parameter „Effizienz“ als Quotient aus zurückgelegter Wegstrecke pro Minute und der mittleren Herzfrequenz unter der Belastung $\left(\mathrm{E}=\mathrm{S} / 6 / \mathrm{f}_{\mathrm{C}}\right)$ als ein objektiver Parameter zur Beurteilung der Leistungsfähigkeit. Er beschreibt die Wegstrecke, die im „steady state“ pro Herzschlag zurückgelegt wird.

Für Patienten mit und ohne $\beta$-Blocker-Therapie wurden die Herzfrequenzen und die Gehstrecken gesondert berechnet und in der - Tab. 3 zusammengestellt.

\section{Statistische Methoden}

Für die einzelnen Gruppen wurden unter Verwendung des Statistikprogramms SPSS Mittelwerte, Standardabweichungen, Median und die Extremwerte berechnet und Boxplots angefertigt. Veränderungen der Testergebnisse zu Beginn und am Ende der Rehamaßnahme wurden mit dem $t$-Test für gepaarte Stichproben auf ihre Signifikanz geprüft. Als Signifikanzschwelle für die Wahrscheinlichkeit für die Nullhypothese wurde $\mathrm{p}<0,05$ angenommen [15].

\section{Ergebnisse}

Wiederholungen des 6 MWT zu Beginn und Ende des Rehaaufenthaltes, Verhalten der Wegstrecken Der 6 MWT zu Beginn und am Ende des Rehabilitationsaufenthaltes führten im 1. Eingangstest (E1) zu einer Gehstrecke (SE1) von $416 \pm 63 \mathrm{~m}$ und in der Wiederholung des Eingangstests (E2) zu einer signifikanten $(p<0,05)$ Steigerung der Gehstrecke auf $454 \pm 71 \mathrm{~m}$ (SE2). Das entspricht einem mittleren Zugewinn von $38,4 \pm 26,2 \mathrm{~m}$ oder 9,3 $\pm 6,2 \%$ ( $\bullet$ Tab. $\mathbf{1}$ ). Im ersten Eingangstest wurden im Mittel 80,8 \pm 13,6\% des Sollwertes erreicht und im 2 . Test eine Steigerung im Mittel auf $88,3 \pm 15,7 \%$ Soll.

Am Ende des Rehaaufenthaltes legten die Patienten im 1. Abschlusstest (A1) eine Strecke (SA1) von $438 \pm 58 \mathrm{~m}$ zurück und in der Wiederholung (A2) ebenfalls eine signifikant $(p<0,01)$ grö-

\begin{tabular}{lllllll}
\multicolumn{2}{l}{ 1. Eingangstest } & \multicolumn{5}{l}{ 2. Eingangstest } \\
& SE1 & $\mathbf{f C E} 1$ & EE1 & SE2 & fCE2 & EE2 \\
& $(\mathbf{m})$ & $(\mathbf{1} / \mathbf{m i n})$ & $\mathbf{( m / H S )}$ & $\mathbf{( m )}$ & $\mathbf{( 1 / m i n )}$ & $\mathbf{( m / H S )}$ \\
\hline MW & 415,9 & 104,7 & 0,673 & $454,3^{*}$ & $106,3 \mathbf{n . s .}$ & 0,726 \\
\hline STD & 62,7 & 15,7 & 0,120 & 71,4 & 17,4 & 0,170 \\
\hline Med & 425,0 & 106,5 & 0,679 & 460,0 & 107,0 & 0,718 \\
\hline Min & 313,0 & 65,0 & 0,399 & 330,0 & 67,0 & 0,404 \\
\hline Max & 520,0 & 130,7 & 0,893 & 550,0 & 139,7 & 0,967 \\
\hline
\end{tabular}

Tab. 1 Vergleich der Wiederholungstestungen des 6 MWT zu Beginn des Rehaaufenthaltes $(n=35)$.

${ }^{*} \mathrm{p}<0.05 ;$ n.s. $=$ nicht signifikant 


\begin{tabular}{|c|c|c|c|c|c|c|}
\hline & \multicolumn{3}{|c|}{ 1. Abschlusstest } & \multicolumn{3}{|c|}{ 2. Abschlusstest } \\
\hline & $\begin{array}{l}\text { SA } 1 \\
\text { (m) }\end{array}$ & $\begin{array}{l}\text { fCA } 1 \\
(1 / \mathrm{min})\end{array}$ & $\begin{array}{l}\text { EA1 } \\
(\mathrm{m} / \mathrm{HS})\end{array}$ & $\begin{array}{l}\mathrm{SA2} \\
\text { (m) }\end{array}$ & $\begin{array}{l}\text { fCA2 } \\
\text { (1/min) }\end{array}$ & $\begin{array}{l}\text { EA2 } \\
(\mathrm{m} / \mathrm{HS})\end{array}$ \\
\hline MW & 437,9 & 106,3 & 0,689 & $473,2 * *$ & 108,6 n.s. & 0,730 \\
\hline \pm STD & 58,4 & 17,4 & 0,085 & 56,6 & 13,3 & 0,137 \\
\hline Med & 438,0 & 104,3 & 0,687 & 485,0 & 105,3 & 0,735 \\
\hline Min & 300,0 & 79,7 & 0,434 & 320,0 & 78,0 & 0,525 \\
\hline Max & 550,0 & 133,7 & 0,870 & 570,0 & 137,3 & 0,915 \\
\hline
\end{tabular}

Tab. 2 Vergleich der Wiederholungstestungen des 6 MWT am Ende des Rehaaufenthaltes $(n=35)$.

${ }^{* *} \mathrm{p}<0.01 ;$ n.s. $=$ nicht signifikant

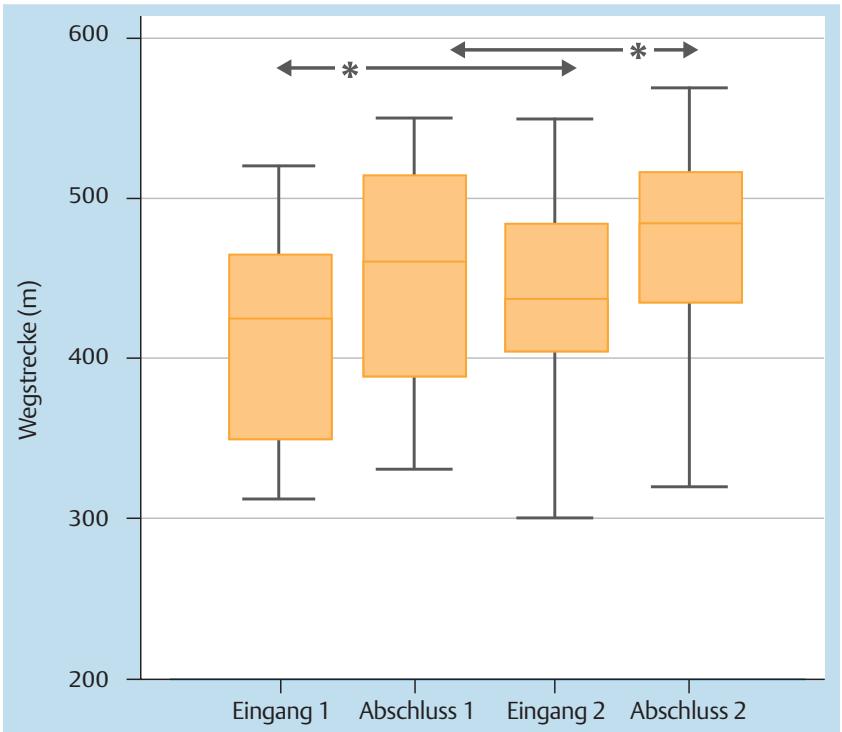

Abb. 1 Verhalten der Wegstrecken in den Eingangs- und Abschlusstestungen und bei deren Wiederholung.

ßere Wegstrecke von (SA2) $473 \pm 57 \mathrm{~m}$ entsprechend einem $\mathrm{Zu}$ gewinn von $35 \pm 26 \mathrm{~m}(8,3 \pm 6,4 \%$ ( Tab. 2, $\bullet$ Abb. 1).

Die Wegstrecken entsprechen $85 \% \pm 13,5 \%$ des Sollwertes im ersten Abschlusstest und in der Wiederholung 91,9 $\pm 13,5 \%$ $(\bullet$ Abb. 2).

Die Zugewinne in beiden Vergleichstests bei Antritt und am Ende der Rehamaßnahme waren damit nahezu identisch. Im 1. Abschlusstest wurde mit $438 \pm 58$ m eine Wegstrecke zurückgelegt, die im Mittel um 22,0 \pm 41 m länger war, als die Wegstrecke im 1 . Eingangstest. In der Wiederholung unterschieden sich die Wegstrecken um 19,0 $\pm 42 \mathrm{~m}$. Damit beträgt der Zugewinn durch die Testwiederholungen im Mittel $+9,3 \pm 6,2 \%$ bzw. $+8,4 \pm 6,4 \%$ der Wegstrecke des 1 . Tests.

Der Zugewinn am Ende der Rehamaßnahme entspricht dem Mittelwert aus den beiden Eingangstestungen.

\section{Verhalten der Herzfrequenz}

Die mittleren Herzfrequenzen von 104,7 $\pm 15,7$ Schläge/min (fCE1) und 106,3 $\pm 17,4$ Schläge/min (fCE2) in den beiden Eingangstesten unterschieden sich nicht signifikant voneinander. Dies gilt auch für die Herzfrequenz in den beiden Abschlusstesten mit 106,3 $\pm 17,4$ (fCA1) bzw. 108,6 $\pm 13,23$ Schläge/min (fCA2) (๑ Abb. 3).

Bei Ausgangsherzfrequenzen in den vier Gehtesten von 81 bis $85 \mathrm{HS} / \mathrm{min}$ führte die Gehbelastung im Mittel um Steigerungen der Herzfrequenz um $23 \pm 9,0 \mathrm{HS} / \mathrm{min}$.

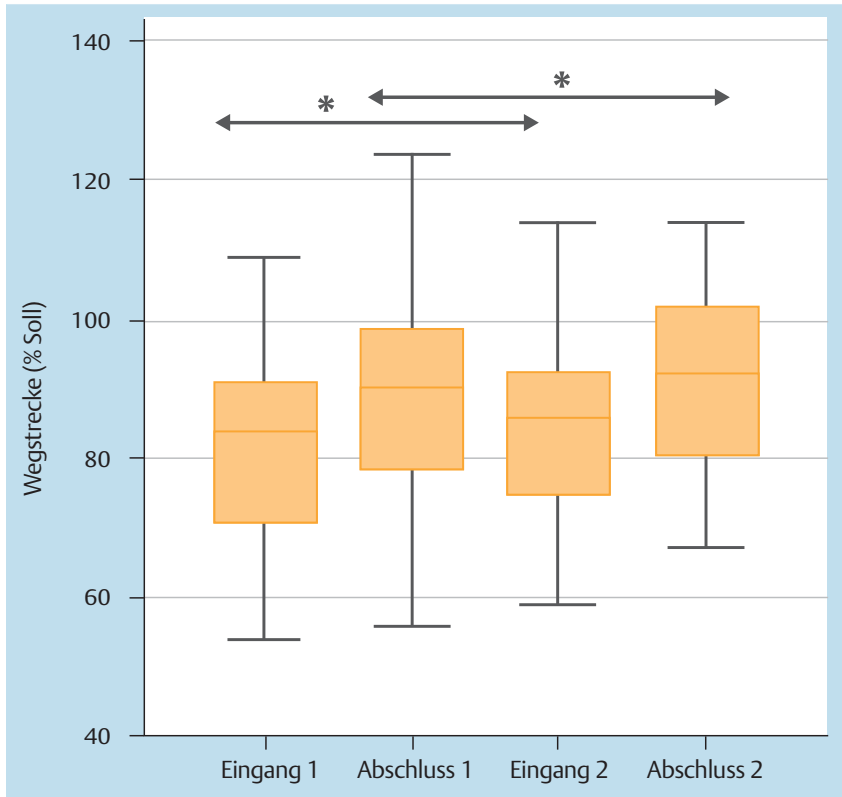

Abb. 2 Verhalten der Wegstrecken in \% des Referenzwertes in den Eingangs- und Abschlusstestungen und bei deren Wiederholung.

\section{Verhalten der Effizienz}

Die Effizienzen als Maß für die körperliche Belastung waren in den beiden 1. Testungen zu Beginn (EE1) und zum Abschluss (EA1) der Rehamaßnahme nicht signifikant verschieden $(0,673$ $\pm 0,129$ bzw. 0,689 $\pm 0,085 \mathrm{~m} / \mathrm{HS}$ ). Auch der Zugewinn an Effizienz in der Wiederholung des Eingangstests (EE2) $(0,053$ $\pm 0,061 \mathrm{~m} / \mathrm{HS}$ ) war vergleichbar mit der Verbesserung der Effizienz in der Wiederholung des Abschlusstests (EA2) $(0,042$ $\pm 0,047 \mathrm{~m} / \mathrm{HS}$ ). ( Abb. 4 u. 6).

\section{Verhalten der Sauerstoffsättigung}

Die Gehtests zum Eingang und Abschluss der Reha führten nur zu geringen, jedoch signifikanten Verminderungen in der Sauerstoffsättigung ( $\bullet$ Abb. 4).

Bei einer mittleren Ausgangsherzfrequenz von 81,0 \pm 12,4 Herzschlägen/min betrug die Sauerstoffsättigung von dem ersten Eingangstest $94,7 \pm 2,2 \%$ und fiel in der 6 . Testminute bis auf 93,2 $\pm 3,4 \%$ ab $(p<0,05)$. In der Wiederholung fiel die $\mathrm{O}_{2}$-Sättigung ausgehend von $95,1 \pm 1,6$ auf $93,3 \pm 3,3 \%$ ab $(p<0,01)$. Im ersten Abschlusstest betrug die $\mathrm{O}_{2}$-Sättigung bei einer mittleren Herzfrequenz von $83,1 \pm 12$,8 Herzschlägen pro Minute $94,6 \pm 1,8 \%$ und fiel in der 6 . Testminute bis auf $92,2 \pm 3,6 \%$ ab $(p<0,001)$. In der Wiederholung des Abschlusstests fiel die $\mathrm{O}_{2}$-Sättigung in der 6. Minute von $95,2 \pm 3,5$ auf $92,6 \pm 3,3 \%$ ab $(\mathrm{p}<0,001)$. 


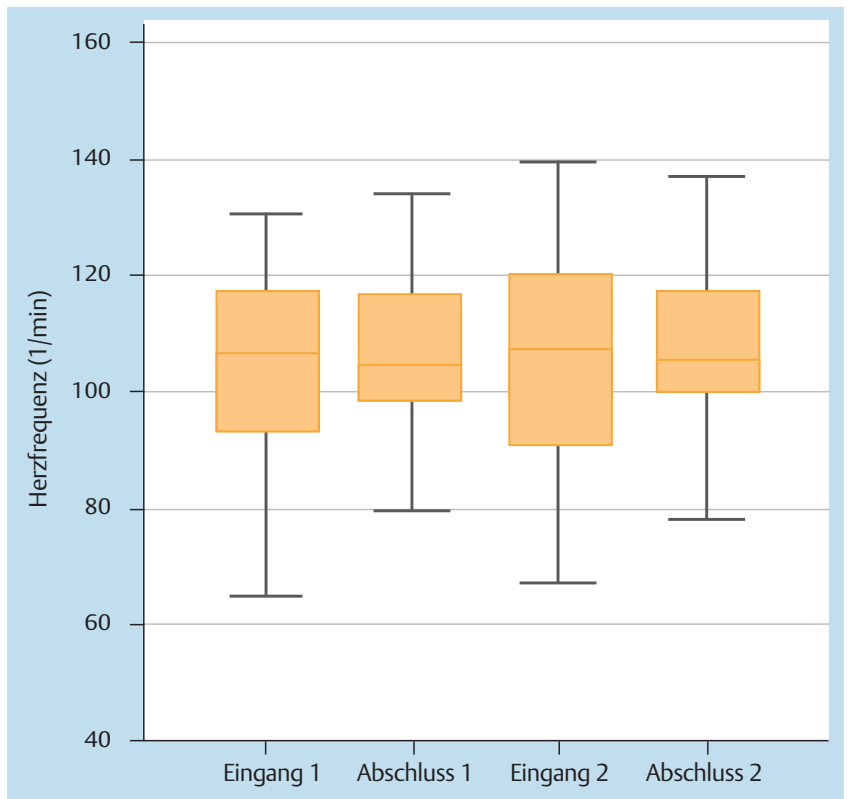

Abb. 3 Verhalten der Herzfrequenzen in den Eingangs- und Abschlusstestungen und bei deren Wiederholung.

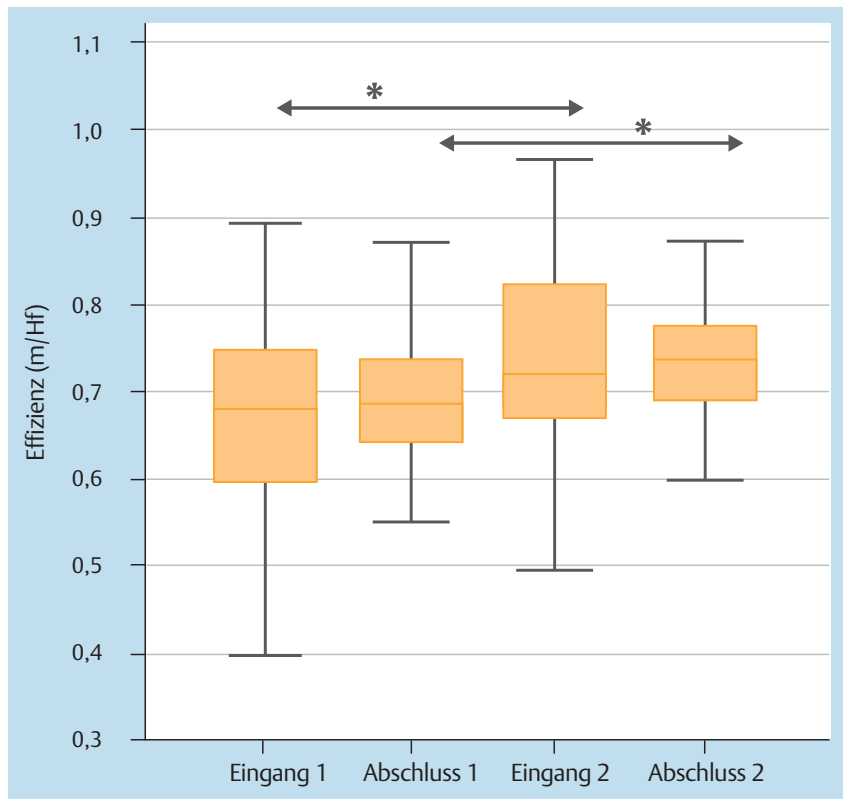

Abb. 4 Verhalten der Effizienz in den Eingangs- und Abschlusstestungen und bei deren Wiederholung.

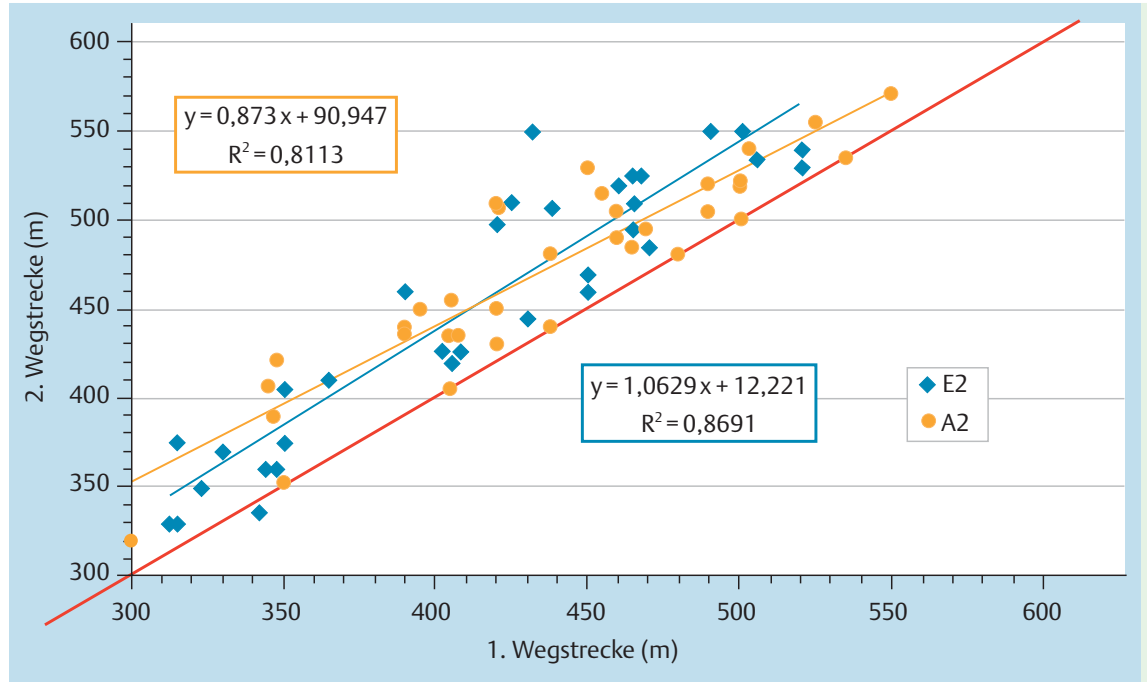

Abb. 5 Korrelation der Wegstrecken (Weg) in der Wiederholung der Eingangstestung (E2, blau) und der Abschlusstestung (A2, orange).

\section{Korrelation von Wegstreckenzunahme und Pulsfrequenzanstieg}

Die Zunahmen der Wegstrecken in der Wiederholung des Eingangs- und Abschlusstests beruhen auf einer erhöhten Belastung mit konsekutivem Pulsfrequenzanstieg gegenüber den ersten Tests. Die Korrelationen von Weg $(S)$ und Herzfrequenz können beschrieben werden mit den Funktionen $S=0,035 x+89,7$ für die Eingangstestungen und $S=0,046 x+86,4$ für die Abschlusstestungen $(\bullet$ Abb. 5).

Belastet sich ein Patient in der Testwiederholung mehr als im 1.Test, so ist seine Herzfrequenz höher als im 1. Test. Eine um im Mittel um 1 Herzschlag/min höhere Herzfrequenz korreliert mit einer Zunahme der Gehstrecke um durchschnittlich $25 \mathrm{~m}$.

\section{Einfluss der $\beta$-Blocker-Therapie}

Die Herzfrequenzen der Patienten mit und ohne Einnahme von $\beta$-Blockern unterschieden sich weder unter den Ausgangswerten (Stehen) noch während der 6-minütigen Gehbelastung
( $\odot$ Tab. 3). $\beta$-Blocker-Patienten legten sowohl in den Eingangsals auch in den Abschlusstesten etwa $15 \mathrm{~m}$ längere Wegstrecken zurück als die Patienten ohne $\beta$-Blocker-Therapie. Die Unterschiede erreichten jedoch nicht das Signifikanzniveau von $\mathrm{p}<0,05$. Auch die Zugewinne durch die Wiederholung des Eingangs- und des Abschlusstests waren vergleichbar mit den Patienten ohne $\beta$-Blocker-Therapie.

Auch die Effizienzen (E) der Patienten mit $\beta$-Blockern waren mit Werten zwischen $0,693 \pm 0,095 \mathrm{~m} / \mathrm{HS}$ im 1 . Eingangstest und $0,748 \pm 0,066 \mathrm{~m} / \mathrm{HS}$ im 2 . Abschusstest tendenziell etwas höher als bei den übrigen Patienten.

\section{Diskussion}

In den Leitlinien der ATS [3] für den 6 MWT wird eine Wiederholung des Tests erwogen, da ein Lerneffekt mit einer Ökonomisierung der 6-minütigen Belastung eintritt und damit ein längerer 


\begin{tabular}{|c|c|c|c|c|c|c|c|c|}
\hline & $\begin{array}{l}\text { Ruhe vor E1, } \\
\text { fCE1 (HS/min) }\end{array}$ & $\begin{array}{l}\text { Ruhe vor E2, } \\
\text { fCE2 (HS/min) }\end{array}$ & $\begin{array}{l}\text { Ruhe vor A1, } \\
\text { fCA1 (HS/min) }\end{array}$ & $\begin{array}{l}\text { Ruhe vor A2, } \\
\text { fCA2 (HS/min) }\end{array}$ & $\begin{array}{l}\text { Weg } \\
\text { SE1 (m) }\end{array}$ & $\begin{array}{l}\text { Weg } \\
\text { SE2 (m) }\end{array}$ & $\begin{array}{l}\text { Weg } \\
\text { SA1 (m) }\end{array}$ & $\begin{array}{l}\text { Weg } \\
\text { SA2 (m) }\end{array}$ \\
\hline mit $\beta$-Blocker & $81,7 \pm 12,7$ & $84,9 \pm 12,1$ & $83,0 \pm 15,0$ & $81,8 \pm 10,1$ & & & & \\
\hline \multirow[t]{2}{*}{ ohne $\beta$-Blocker } & $81,0 \pm 12,4$ & $\mathbf{8 4 , 5} \pm 13,6$ & $83,7 \pm 12,0$ & $85,6 \pm 10,5$ & & & & \\
\hline & Mittelwert fCE1 & Mittelwert fCE2 & Mittelwert fCE1 & Mittelwert fCA2 & & & & \\
\hline mit $\beta$-Blocker & $\mathbf{1 0 4 , 0} \pm 18,9$ & $106,3 \pm 17,4$ & $103,9 \pm 11,1$ & $\mathbf{1 0 8 , 6} \pm 13,2$ & $425 \pm 56,9$ & $475 \pm 71$ & $444 \pm 42,7$ & $489 \pm 53,5$ \\
\hline ohne $\beta$-Blocker & $104, \mathbf{4} \pm 15,7$ & $108,3 \pm 20,5$ & $106,8 \pm 17,4$ & $109,3 \pm 10,7$ & $407 \pm 83,3$ & $446 \pm 92$ & $427 \pm 84,5$ & $464 \pm 86,8$ \\
\hline
\end{tabular}

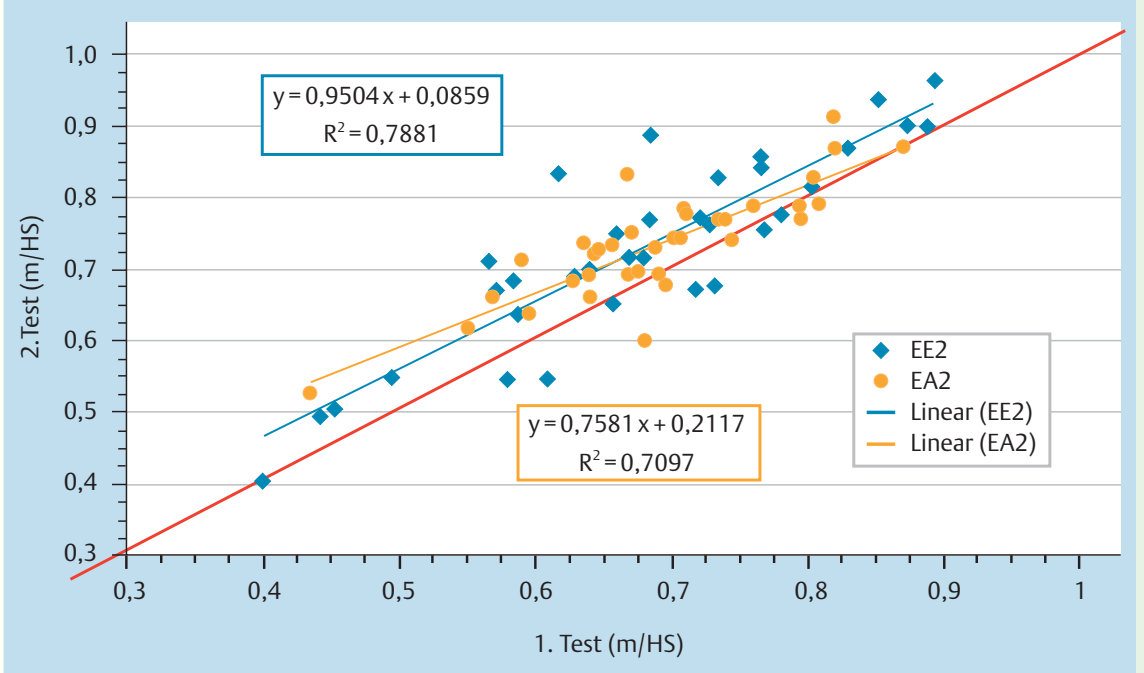

Abb. 6 Korrelation der Effizienzen (E) in der Wiederholung der Eingangstestung (EE2, blau) und der Abschlusstestung (EA2, orange).

Weg zurückgelegt werden kann. Ein Lerneffekt würde eine Wegstreckenzunahme im Abschlusstest als Erfolg einer Rehamaßnahme überbewerten. Die Wiederholungen finden in der klinischen Routine jedoch wenig Akzeptanz. Zeit- und Personalmangel werden nämlich ständig größer. In einer aktuellen Übersichtsarbeit über den 6 MWT wird die Wiederholung des Tests bzw. ein Übungslauf nicht ausdrücklich gefordert [1]. In einer Gruppe von 35 Patienten wurde der Gehtest zu Beginn und am Abschluss der Rehamaßnahme jeweils zweimal durchgeführt. Die mittlere Gehstrecke steigerte sich bei der Wiederholung des Eingangstests um 38,4 $\pm 26,2 \mathrm{~m}(9,3 \pm 6,2 \%)$ und im Abschlusstest vergleichbar mit dem Zugewinn im Eingangstest um $35 \pm 26$ m (8,4 $\pm 6,6 \%$ ). Die Wiederholung des Eingangstests und des Abschlusstests führten somit zu vergleichbaren Steigerungen der Wegstrecke. Es macht keinen Unterschied, ob man das Ergebnis des ersten Eingangstests mit dem des ersten Abschlusstests miteinander vergleicht oder ob man jeweils die beiden Wiederholungstests miteinander vergleicht. In beiden Fällen lag der Zugewinn an Gehstrecke nach der Rehamaßnahme im Mittel bei $20 \mathrm{~m}$. Der Lerneffekt, der durch die Wiederholung des Eingangstests gemessen wurde, entspricht im Mittel 8,9 \pm 6,3\% und scheint nach unseren Ergebnissen nach 3 bis 4 Wochen zum Zeitpunkt der Abschlussuntersuchung verloren zu sein.

Der Zugewinn in der Wiederholung der beiden Tests am Beginn und am Ende der Rehamaßnahme war dabei unabhängig von der Länge der Eingangwegstrecke. Somit kann bei Verzicht auf eine Testwiederholung durch Addition von 9\% der Teststrecke das Ergebnis einer Testwiederholung abgeschätzt werden.

Eine Steigerung der Wegstrecke nach Ablauf der Rehamaßnahme als Ausdruck einer Verbesserung der körperlichen Leistungsfähigkeit zeigte sich in einer früheren Arbeit insbesondere bei den Patienten, die eine geringe Eingangswegstrecke aufwiesen [14].
Diese rekrutierten sich vornehmlich aus älteren Patienten. Ab einer Eingangswegstrecke von mehr als $360 \mathrm{~m}$ war im Mittel keine weitere signifikante Steigerung mehr zu erreichen. 95\% der Patienten konnten beide Gehtests komplett ausführen. Komplikationen, die ein notfallmäßiges Eingreifen nötig gemacht hätten, wurden nicht registriert. Ursache für den Abbruch waren körperliche Erschöpfung oder orthopädische Beschwerden im Bereich der Wirbelsäule und der Beine. Nur 24\% der Patienten erreichten am Ende des Rehaaufenthaltes eine relevante Zunahme der Gehstrecke von mehr als $54 \mathrm{~m}$, bei $54 \%$ betrug der Wegstreckenzugewinn zwischen 1 und 53 m, während $20 \%$ der Patienten im Abschlusstest eine kürzere Gehstrecke zurücklegten als im Eingangstest. Die kürzere Wegstrecke war regelmäßig mit einer niedrigeren Herzfrequenz verbunden. Bei 33\% der Patienten war die mittlere Herzfrequenz im Abschlusstest niedriger als im Eingangstest. Die Ergebnisse sind ein Zeichen dafür, dass sich ein bedeutender Teil der Probanden im Abschlusstest weniger belasteten als im Eingangstest. Der in der früheren Arbeit vorgestellte Parameter Effizienz (E) erlaubt eine Objektivierung des Testergebnisses, wenn kein signifikanter Wegstreckenzugewinn auf Grund einer eingeschränkten Mitarbeit am Ende einer Rehamaßnahme erzielt wurde.

Bei gesunden Probanden besteht eine unabhängige Korrelation zwischen der Gehstrecke und dem Lebensalter, der Körpergröße, dem Bauchumfang und dem Körpergewicht [16]. Mit zunehmendem Lebensalter vermindern sich die Skelettmuskelmasse und die Kraft, beide Faktoren tragen zur Gehstreckenverminderung älterer Menschen bei $[17,18]$. Zunehmende Körperlänge ist verbunden mit einer größeren Schrittlänge, die das Gehen effizienter macht. Korrigiert man um diese Faktoren, so ist die Gehstrecke älterer Männer und Frauen kaum unterschiedlich [7]. Übergewicht erhöht die zu erbringende Leistung und führt damit zur 
Gehstreckenverkürzung. Probanden mit einem BMI > 30 erreichen bis zu 85\% der Gehstrecke normalgewichtiger Personen. Der Bauchumfang erwies sich als ein besseres Maß als der BMI [7].

Die Gehstrecken der Patienten bewegten sich in einer früheren Untersuchung [14] bei $353 \mathrm{~m}$ im Mittel bei 80\% des Referenzwertes nach Enright [12] und verbesserten sich zum Abschluss der Rehabilitation im Mittel um 19 m, also weitaus weniger als 54 m, die für eine relevante Leistungssteigerung gefordert werden. Wie auch von früheren Untersuchern beschrieben, verbesserte sich die Gehstrecke insbesondere bei den Patienten mit besonders stark eingeschränkter Eingangsgehstrecke. Unsere Ergebnisse sind vergleichbar mit denen von Halle et al. 2008 [19], die über signifikante Gehstreckenzunahmen bei Patienten mit unterschiedlichen Erkrankungen berichten, die im Ausgangstest mittlere Wegstrecken zwischen 250 und $300 \mathrm{~m}$ erreichten. Bei Patienten mit weniger ausgeprägten Erkrankungen und annähernd normaler Gehstrecke bzw. Leistungsfähigkeit sind weniger deutliche Steigerungen während der Rehabilitationsmaßnahme zu beobachten. Die Leistungen, die von den Patienten bei einer mittleren Gehgeschwindigkeit von 3,5 km/h aufzubringen sind, bewegen sich nach Angaben von Löllgen in der Größenordnung von bis zu 50 Watt und entsprechen einer Sauerstoffaufnahme von weniger als $1,0 \mathrm{~L} / \mathrm{min}$ [20].

Beobachtete mittlere Ganggeschwindigkeiten von gesunden Männern betragen $82 \mathrm{~m} / \mathrm{min}$ und $74 \mathrm{~m} / \mathrm{min}$ bei Frauen [21]. Sie entsprechen etwa den hier verwendeten Sollwerten von Enright. Bei Geschwindigkeiten über $100 \mathrm{~m} / \mathrm{min}$ kann man wahlweise gehen oder laufen. Die Übergangsgeschwindigkeit vom Gehen zum Laufen beträgt bei Männern durchschnittlich $113 \mathrm{~m} / \mathrm{min}$, bei langbeinigen Männer liegt der Übergang höher [22]. Bei der bevorzugten Gangart beträgt die Sauerstoffaufnahme von Senioren zwischen 60 und 80 Jahren 12,1 ml/kg/min [21]. Das entspricht bei einem 75-Jährigen $48 \%$ der maximalen $\mathrm{O}_{2}$-Aufnahme [23]. Der RQ beträgt dabei unabhängig von der Altersgruppe 0,85 und befindet sich damit im aeroben Bereich. Bei hoher Ganggeschwindigkeit beträgt die $\mathrm{O}_{2}$-Aufnahme bei jungen Erwachsenen $19,6 \mathrm{ml} / \mathrm{kg} / \mathrm{min}$ [21], bei Senioren $15,0 \mathrm{ml} / \mathrm{kg} / \mathrm{min}$. Die Unterschiede in der Geschwindigkeit betragen dabei $106 \mathrm{~m} / \mathrm{min} \mathrm{zu}$ $90 \mathrm{~m} / \mathrm{min}$. Die respiratorische Gasaustauschrate beträgt bei $\mathrm{Er}-$ wachsenen und Senioren 0,92 für den Übergang zum anaeroben Stoffwechsel [21].

In der vorliegenden Untersuchung lagen die Eingangswegstrecken im 1. Test im Mittel bei $416 \mathrm{~m}$ und im 1. Abschlusstest bei 438 m. Die Wegstrecken entsprechen 81 \% bzw. 85\% des Sollwertes. Dabei wurden im Mittel Herzfrequenzen von $107 \mathrm{HS} / \mathrm{s}$ bei Patienten ohne $\beta$-Blocker und $104 \mathrm{HS} / \mathrm{s}$ bei Patienten mit $\beta$-Blockern erreicht.

Sollte sich ein Patient in der Eingangsuntersuchung nicht erwartungsgemäß belastet haben, so gibt die Effizienz bereits Auskunft über seine Leistungsfähigkeit. Bei der linearen Korrelation von Herzfrequenz und Gehgeschwindigkeit in dem hier vorliegenden Leistungsbereich von 50 bis100 Watt [20] ist die Steigung der Kennlinie und damit die Effizienz konstant.

Zur Berechnung der Effizienz aus der mittleren Herzfrequenz braucht nicht ausdrücklich auf die Gewinnung der validen Ausgangs- oder Ruheherzfrequenz geachtet werden. Die Probanden sollten jedoch vor Beginn des Tests ausreichend Zeit zur Ruheadaptation und eine Sitzgelegenheit haben, während sie mit dem Test vertraut gemacht und mit dem Pulsoximeter ausgestattet werden. Ruhefrequenzen von 70 bis $80 \mathrm{HS} /$ min erscheinen repräsentativ und dem Gesundheits- und Trainingszustand der Pa- tienten zu entsprechen. Die Berechnung der Effizienz aus Differenz zwischen dem Ruhepuls und dem Pulsanstieg unter der Belastung wäre sicherlich sinnvoll, jedoch erwies sich die Gewinnung der Ruheherzfrequenz als problematisch.

Ist der Ausgangspuls bereits erhöht, wird die Pulszunahme durch die Belastung zu gering bestimmt und der Effekt der Extrapolation fällt zu hoch aus, da die Kennlinie zu flach verläuft. Messabstände von 30 Sekunden für die Herzfrequenz und die Sauerstoffsättigung sind ausreichend und ergeben 12 Messwerte für die Berechnung der mittleren Frequenz unter Belastung und gleicht mögliche Unterschiede in der Belastung im Testsverlauf weitgehend aus. Daher sollte besonders darauf geachtet werden, dass die Patienten vor dem Test ausreichend Zeit zur Adaptation an Ruhebedingungen finden.

\section{Schlussfolgerungen \\ $\nabla$}

Sowohl die Wiederholung des Eingangstests als auch die Wiederholung des Abschlusstests ergaben vergleichbare mittlere Wegstreckenzugewinne. Damit ist ein Lernerfolg zwischen der 1. Eingangsuntersuchung und der 1. Abschlussuntersuchung nicht mehr nachweisbar. Damit beruht der Zugewinn an 6 MWD nach 3-4 Wochen Rehamaßnahme nicht auf dem Lerneffekt, sondern auf den Verbesserungen der körperlichen Leistung durch das Kraft- und Ausdauertraining. Insgesamt führte die Rehamaßnahme zu einer mittleren Verbesserung der Wegstrecke um $22 \mathrm{~m}$. Die Ergebnisse von Patienten mit $\beta$-Blocker-Therapie waren vergleichbar mit denen der übrigen Patienten. In der klinischen Routineuntersuchung kann damit zur Einsparung von Zeit und Personal auf eine Wiederholung der beiden Testungen durchaus verzichtet werden, wenn man den Erfolg einer Rehamaßnahme beurteilen will. Die Größe des Wegstreckenzugewinns (Lerneffekt und größere Motivation) lässt sich mit etwa 9\% der Testwegstrecke abschätzen. Die Effizienz, E, ist eine ergänzende, weitgehend objektive Messgröße für die körperliche Leistungsfähigkeit und sollte zusätzlich zur Betrachtung der Wegstrecke in die Begutachtung einfließen.

\section{Interessenkonflikte \\ $\nabla$}

Die Autoren geben an, dass kein Interessenkonflikt besteht.

\section{Literatur}

1 Salzman SH. The 6-min walk test. Clinical and research role, technique, coding and reimbursement. Chest 2009; 35: 1345-1352

2 Guralnik J, Branch L, Cummings S et al. Physical performance measures in aging research. J Gerontol Med Sci 1989; 44: M141 - M146

3 American Thoracic Society. ATS Statement: Guidelines for six-minute walk test. Official statement of the american thoracic society. Am J Respir Crit Care Med 2002; 166: 111-117

4 Roca J, Whipp BJ. Clinical Exercise testing. Eur Respir Monograph 1997; 2: $1-164$

5 Hien P, Morr H. 6-Minuten-Gehtest in der pneumologischen und kardiologischen Diagnostik: Methodik, Bedeutung, Grenzen. Pneumologie 2002; 56: $558-566$

6 Knox AJ, Morrisom JFJ, Muers MF. Reproducibility of walking test results in chronic obstructive disease. Thorax 1988; 43: 388 - 392

7 Enright PL, McBurnie MA, Bittner $V$ et al. The 6-min walk test. A quick measure of functional status in elderly adults. Chest 2003; 123: 387 398

8 Deutsche Gesellschaft für Pneumologie. Empfehlungen zur Durchführung und Bewertung von Belastungsuntersuchungen in der Pneumologie. Pneumologie 1998; 52: 225-231 
9 Troosters T, Gosselink R, Decramer M. Six minutes walking distance in healthy elderly subjects. Eur Respir J 1999; 14: 270-274

10 Peeters $P$, Mets T. The 6 minute walk as an appropriate exercise test in elderly patients with chronic heart failure. Gerontol 1996; 51A: M147-M151

11 Nici L, Donner C, Wouters E et al. American thoracic society/european respiratory society statement on pulmonary rehabilitation. Am J Respir Crit Care Med 2009; 173: 1390-1413

12 Enright PL, Sherril DL. Reference equations for the six-minutes walk in healthy adults. Am J Respir Crit Care Med 1998; 158: 1384-1387

13 Redelmeier DA, Bayoumi AM, Goldstein RS et al. Interpreting small differences in functional status: the six minutes walk test in chronic lung disease patients. Am J Respir Crit Care Med 1997; 155: 1278 - 1282

14 Marek W, Marek E, Vogel P et al. Ein numerisches Verfahren zur Objektivierung der körperlichen Leistungsfähigkeit im Rahmen eines stationären Rehabilitationsaufenthaltes mittels 6-Minuten Gehtest. Pneumologie 2008; 62: 643-654

15 Sachs L. Angewandte Statistik, Anwendung statistischer Methoden. 7. Aufl. Berlin, Heidelberg, New York: Springer, 1992
16 Hirsch GL, Fried LP, Harris $T$ et al. Correlates of performence-based measures of muscle function in the elderly: The cardiovascular health study. J Gerontol Med Sci 1997; 52A : M192 - M200

17 Fleg JL, Lakatta EG. Role of muscle loss in the age-associated reduction in VO2max. J Am Geriatr Soc 1988; 46: 706 - 711

18 Tolep K, Kelsen SG. Effect of aging on respiratory skeletal muscles. Clin Chest Med 1993; 3: 363 - 378

19 Halle M, Heitmann R, Kenn K et al. Bedeutung und Methodik von körperlichem Training bei COPD. Pneumologie 2009; 62: 209-225

20 Löllgen $H$. Kardiopulmonale Funktionsdiagnostik. 3. Auflage. Wehr/Baden: Novartis Pharma GmbH, 2000

21 Waters RL, Lunsford BR, Perry R et al. Energy-speed relationship of walking: standard tables. J Orthop Res 1988; 6: 215 - 222

22 Thorstensson A, Roberthson HR. Adaptations to changing speed in human locomation: speed of transition between walking and running. Acta Physiol Scand 1987; 131: $211-214$

23 Astrand A, Astrand I, Hallback I et al. Reduction of maximal oxygen uptake with age. J Appl Physiol 1973; 35: 649-654 